\title{
Diversité Et Activité Antimicrobienne Des Plantes Impliquées Dans Le Traitement Des Affections Dermatologiques Chez Les Peul Et Les Wolof Du Ferlo Nord (Sénégal)
}

\section{Bétémondji Désiré Diatta,}

Laboratoire de Botanique, Département de Botanique et Géologie, IFAN Cheikh. A. Diop/UCAD « Environnement, Santé et Société », Faculté de Médecine Pharmacie Odontostomatologie UCAD

Ousmane Niass,

Laboratoire de chimie analytique Faculté de Médecine Pharmacie Odontostomatologie à l'université Cheikh Anta Diop de Dakar, Afrique

\section{Mathieu Gueye,}

Département de Botanique et Géologie, Laboratoire de Botanique, IFAN

Cheikh. A. Diop/UCAD Sénégal

\section{Emeline Houël,}

CNRS, UMR EcoFoG, AgroParisTech, Cirad, INRA, Université de Guyane,

\section{Cayenne, France}

\section{Gilles Boetsch,}

Directeur de recherche au CNRS, Environnement, santé société, faculté de médecine secteur Nord, Marseille, France

Doi:10.19044/esj.2022.v18n8p73

Submitted: 23 November 2021

Accepted: 28 January 2022

Published: 28 February 2022
Copyright 2022 Author(s)

Under Creative Commons BY-NC-ND 4.0 OPEN ACCESS

Cite As:

Diatta B.D.,Niass O., Gueye M., Houël M., \& Boetsch G.,(2022). Diversité Et Activité Antimicrobienne Des Plantes Impliquées Dans Le Traitement Des Affections Dermatologiques Chez Les Peul Et Les Wolof Du Ferlo Nord (Sénégal) European Scientific Journal, ESJ, 18 (8), 73.

https://doi.org/10.19044/esj.2022.v18n8p73

\section{Résumé}

Ce travail adhère à l'initiative de valorisation des végétaux conduite par la Grande Muraille Verte (GMV) pour faire face à la disparition des végétaux et des savoir-faire végétaux associés. L’objectif de cette étude est de déterminer la diversité végétale impliquée dans le traitement des pathologies dermatologiques au Ferlo (Sénégal) et d’évaluer l'activité antimicrobienne des plantes les plus utilisées. Pour ce faire, un guide d'entretien semi directif a été 
soumis aux enquêtés, afin de recueillir la diversité des plantes utilisées au Ferlo dans le traitement des dermatoses. Des tests de micro dilution ont permis d'évaluer la concentration minimale inhibitrice (CMI) d'extraits de Sept (7) plantes contre une souche fongique (Candida albicans) et une bactérienne (Staphyloccocus aureus). Vingt-huit (28) espèces réparties dans 26 genres et 14 familles ont été recensées comme impliquées dans le traitement des affections dermatologiques. L'écorce est la partie la plus exploitée, puis le latex, tandis que, la décoction, suivie de l'usage direct représentent les modes de préparation les plus fréquents. Treize (13) parmi les plantes proposées sont exclusivement utilisées contre les dermatoses, quatre (4) fréquemment exploitées, et onze (11) occasionnellement recommandées. Widou Thiengoli est la localité où l'on exploite le plus d'espèces suivi de Loughere Thiolly. Leptadenia lancifolia (Schumach.\& Thonn.) Decne. Stereospermum kunthianum Cham. et Sterculia setigera Delile ont été identifiées comme actives contre Candida albicans avec respectivement des CMI de 1,5 mg/ml pour les deux premières et $0,755 \mathrm{mg} / \mathrm{ml}$ pour la troisième. Acacia seyal Delile, Sterculia setigera Delile et Anogeissus leiocarpa (DC) Guill. \& Perr. ont détruit Staphylococcus aureus avec respectivement des CMI de $3 \mathrm{mg} / \mathrm{ml}$ pour les deux premières et $1,5 \mathrm{mg} / \mathrm{ml}$ pour la troisième.

Mots-clés : Ethnobotanique, Affections dermatologiques, Activités antimicrobiennes, Ferlo Nord, Sénégal 


\section{Diversity, Antibacterial And Antifungal Activities of Plants Involved in the Treatment Of Dermatological Conditions in the Peul and Wolof of the North of Ferlo (Senegal)}

\section{Bétémondji Désiré Diatta,}

Laboratoire de Botanique, Département de Botanique et Géologie, IFAN Cheikh. A. Diop/UCAD « Environnement, Santé et Société », Faculté de Médecine Pharmacie Odontostomatologie UCAD

\section{Ousmane Niass,}

Laboratoire de chimie analytique Faculté de Médecine Pharmacie

Odontostomatologie à l'université Cheikh Anta Diop de Dakar, Afrique

Mathieu Gueye,

Département de Botanique et Géologie, Laboratoire de Botanique, IFAN

Cheikh. A. Diop/UCAD Sénégal

\section{Emeline Houël,}

CNRS, UMR EcoFoG, AgroParisTech, Cirad, INRA, Université de Guyane, Cayenne, France

Gilles Boetsch,

Directeur de recherche au CNRS, Environnement, santé société, faculté de médecine secteur Nord, Marseille, France

\section{Abstract}

This work adheres to the plant enhancement initiative led by the Great Green Wall (GGW) to deal with the disappearance of plants and associated plant know-how. The objective of this study is to determine the plant diversity involved in the treatment of dermatological pathologies in Ferlo (Senegal) and to evaluate the antimicrobial activity of the most used plants. To do this, a semi-directive interview guide was submitted to respondents, in order to promote the diversity of plants used at Ferlo in the treatment of dermatoses. Microdilution tests made it possible to evaluate the minimum inhibitory concentration (MIC) of extracts of seven (7) plants against a fungal strain (Candida albicans) and a bacterial strain (Staphyloccocus aureus). Twentyeight (28) species distributed in 26 genera and 14 families have been referenced as involved in the treatment of dermatological conditions. The bark is the most used part, then the latex, while the decoction, followed by direct use, represents the most frequent methods of preparation. Thirteen (13) among the proposed plants are exclusively used against dermatoses, four (4) frequently used, and eleven (11) occasionally recommended. Widou Thiengoli is the locality where the most species are exploited, followed by Loughere Thiolly. Leptadenia lancifolia (Schumach. \& Thonn.) Decne. Stereospermum kunthianum Cham. and Sterculia setigera Delile were administered as active 
agents against Candida albicans with MICs of $1.5 \mathrm{mg} / \mathrm{ml}$ for the first two and $0.755 \mathrm{mg} / \mathrm{ml}$ for the third, respectively. Acacia seyal Delile, Sterculia setigera Delile and Anogeissus leiocarpa (DC) Guill. \& Perr. destroyed Staphylococcus aureus with respectively MICs of $3 \mathrm{mg} / \mathrm{ml}$ for the first two and $1.5 \mathrm{mg} / \mathrm{ml}$ for the third.

Keywords: Ethnobotany, Dermatological conditions, Antimicrobial activities, North Ferlo, Senegal.

\section{Introduction}

La santé et la beauté de la peau constituent une préoccupation constante au Ferlo. Une peau saine, dépourvue d'irruptions, de plaies, d'irrégularités est, symbole de santé, mais aussi d'appartenance à une classe sociale favorisée (Diatta, 2019). Toute altération de la peau, en particulier celle du visage, serait considérée comme dévalorisante et liée à un discrédit moral, voire religieux. Par ailleurs, les affections dermatologiques humaines représentent en zone intertropicale, et notamment au Sénégal un sérieux problème de santé.

Les maladies cutanées sont très présentes au Ferlo notamment dans la commune de Téssékéré Forage et y sont et traditionnellement traitées (Cissé et al., 2016). Malgré la fragilité de son écosystème, la flore locale est diversement utilisée par la population locale (Cissé et al., 2016 ; Niang et al., 2014). La précarité des milieux dans lesquels vivent ces populations, ajoutée aux nombreux travaux physiques qu'elles sont contraintes de réaliser à longueur de journée les expose à plus d'altérations de la peau (Senghor, 2008). Les troubles dermatologiques sont également aujourd'hui aggravés par la multiplication d'infections opportunistes liées au VIH/ SIDA (Mozouloua et al., 2011). Les patients infectés par le VIH, présentent souvent une résistance au traitement par l'antifongique le plus couramment utilisé, le fluconazole (Redding et al., 1994).

De plus, le coût des traitements médicaux n'étant souvent pas à la portée des populations, le recours à la médecine traditionnelle, devient alors incontournable. Si des études antérieures réalisées au Sénégal (Diatta et al., 2013; Cissé et al., 2012) ont permis de recenser une flore abondante impliquée dans le traitement des dermatoses, l'essentiel des espèces proposées dans cette médecine traditionnelle n'ont pas fait l'objet de recherche ni pour leur teneur phytochimique ni pour leurs propriétés pharmacologiques (Togola, 2005).

L'intérêt actuel croissant de l'usage des plantes, notamment médicinales, comme alternative sûre et renouvelable aux molécules synthétiques permet ainsi de légitimer les recherches sur les propriétés des plantes (Rodrigues et al., 2019; Houël et al., 2014 ; Rios et Recio, 2005 ). En effet, il existe 
aujourd'hui sur le marché quelques d'antifongiques connus d'origine végétale ou inspirés par les plantes (Newmann et Cragg, 2016).

C'est pourquoi, l'objectif de cette étude est recueillir les plantes proposée dans le traitement des dermatoses au Ferlo et déterminer l'activité antimicrobienne des espèces les plus citées contre deux souches microbiennes pathogènes.

\section{Méthodologie}

\section{Zone d'étude}

Le Ferlo, situé entre les latitudes $15^{\circ}$ et $16^{\circ} 30$ Nord et les longitudes $13^{\circ} 30$ et $16^{\circ}$ Ouest, couvre une superficie de $70.000 \mathrm{~km}^{2}$ (Miehe, 2007). Il est au Nord du Sénégal et plus précisément entre la vallée du fleuve Sénégal au Nord et à l'Est, le lac de Guiers à l'Ouest et la vallée fossile du Ferlo au Sud. Cette région comporte un climat semi-aride distinctif de la ceinture sahélienne, avec des précipitations annuelles de 300-600 mm/an. L'année est scindée en deux saisons : une brève saison des pluies, de juillet à septembre-octobre et une saison sèche de 8 à 9 mois entre octobre-novembre et juin (Bakhoum, 2013). Les températures, comprises entre 24 et $30^{\circ} \mathrm{C}$, comportent une valeur moyenne de $27,7^{\circ} \mathrm{C}$. La végétation est une steppe avec une strate herbacée importante et des espèces ligneuses de petite taille (arbres et arbustes), en majorité épineuses, et clairsemées (Ndong et al., 2015 ; Niang et al., 2014). D’un point de vue socio-démographique, le Ferlo est habité en majorité par des Peul (90\%) et des Wolof (10\%) (Niang et al., 2014). Les enquêtes ethnobotaniques ont été menées dans les villages de Widou Thiengoli, Téssékéré Forage, Amaly, Labgar, Loughere Thiolly et Barkédji (Ferlo Nord) (Figure 1). La commune de Tessekere Forage comporte la moitié des localités visitées et a une population estimée à 9281 habitants lors du recensement de 2013 (ANSD, 2013).

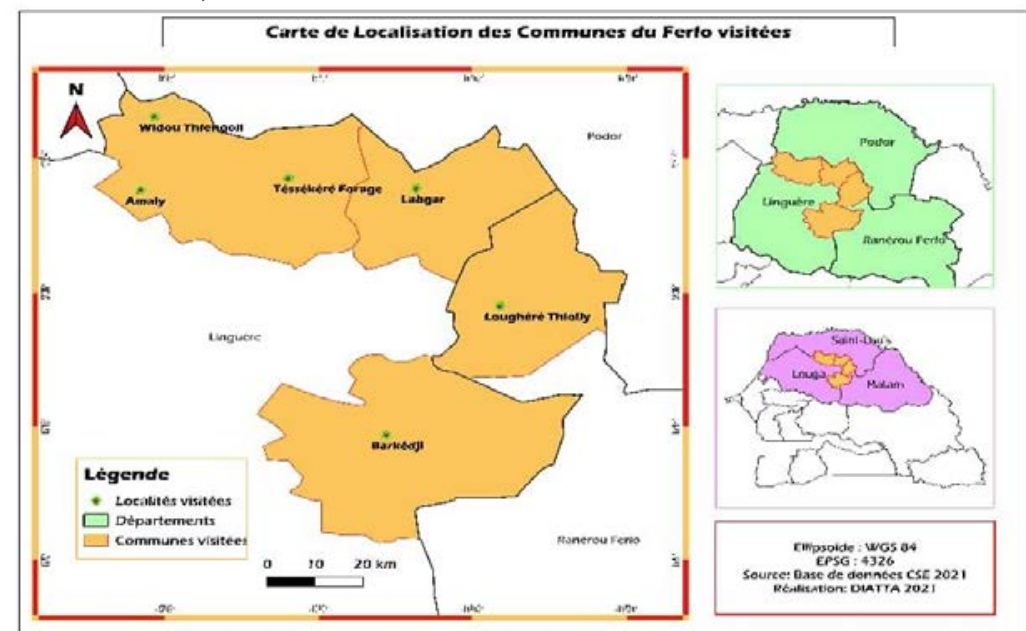

Figure 1 Situation géographique des localités visitées lors de l'enquête au Ferlo. 


\section{Enquêtes ethnobotaniques}

Les enquêtes ont été réalisées selon la technique des entretiens ouverts semi-structurés, grâce à un guide d'entretien élaboré de manière à recueillir tous les noms des plantes ayant une ou des fonctions cosmétiques dans la zone, les parties de plantes utilisées, le mode de préparation, le mode d'utilisation et la partie du corps ciblée. Les conversations anodines et des observations directes ont par ailleurs été exploitées. Seules les plantes utilisées dans les affections dermatologiques sont présentées dans cette étude. Les enquêtes ethnobotaniques ont été réalisées entre 2014 et 2018. Le choix des personnes à interroger s'est fait avec l'aide de la population locale et pour chaque localité, les entretiens ont été poursuivis jusqu’à ce qu'il n’y ait plus de nouvelles espèces citées (saturation). Un interprète possédant une bonne connaissance de la langue locale a été associé aux entretiens et les noms des plantes ont été donnés par les enquêtés eux-mêmes en langue locale peul et wolof. Des sorties de terrain ont ensuite été effectuées pour identifier les espèces indiquées et prélever des échantillons pour la confection d'un d'herbier. L'identification des espèces citées a été faite le plus souvent sur place à l'aide d'ouvrages de référence (Arbonier, 2002 ; Berhaut, 1967), confirmée par comparaison avec des échantillons d'herbier de la collection de l'IFAN. Les noms valides sont ceux du site http://www.worldfloraonline.org/. La cohérence des informations est vérifiée selon la technique d’El Rhaffari et al. (2002). Une information est considérée comme cohérente lorsqu'elle est rapportée au moins deux fois dans deux localités différentes et par des informateurs différents, sinon elle est dite divergente. Seules les informations cohérentes ont été retenues dans le traitement des données.

\section{Le traitement des données}

Le traitement des données a été réalisé par des techniques statistiques descriptives et particulièrement par le calcul de l'indice de fidélité selon la méthode de Begossi (1996) selon la formule suivante : $\mathbf{I F}=\mathbf{I p} / \mathbf{I u} \times \mathbf{1 0 0}$ Avec Ip le nombre d'informateurs ayant affirmé l'emploi d'une espèce précise dans une catégorie d'usage donnée et Iu le nombre total d'informateurs ayant cité la plante dans n’importe quelle catégorie d'usages.

\section{Extraction}

Les plantes retenues pour l'évaluation de l'activité anti-microbienne ont été sélectionnées sur la base de leur nombre de citations et de leur niveau de fidélité. Les plantes ont été récoltées aux environ de 17 heures et pour chaque espèce, environ $500 \mathrm{~g}$ de matériel végétal ont été collectés et séchés à l'ombre à température ambiante, étalés sur des papiers de journal au laboratoire de botanique de l'IFAN. Les parties des plantes utilisées (écorce, feuilles, racines) ont ensuite été réduites en fine poudre par broyage à l'aide 
d'un broyeur motorisé brabender ${ }^{\circledR}$ Duisburg . Pour chaque plante, 50 g de poudre ont été mis à macérer dans des bouteilles en verre avec $500 \mathrm{~mL}$ d'eau à $90^{\circ} \mathrm{C}$ et soumis à extraction pendant une heure (Niass et al., 2015). Le mélange ainsi obtenu est filtré sous vide et la partie aqueuse est concentrée à sec à l'évaporateur rotatif.

\section{Préparation des échantillons}

Les extraits aqueux secs obtenus ont été utilisés pour évaluer l'activité antimicrobienne des extraits sélectionnés. Ces extraits secs ont été dissous dans le diméthyle sulfoxyde (DMSO) (Sreekumar et Hosono, 1998 ) à une concentration de base de $6 \mathrm{mg} / \mathrm{mL}$. Ce sont ces solutions qui sont utilisées pour l'évaluation de l'activité antimicrobienne.

\section{Préparation des antibiotiques}

Les antibiotiques de références utilisées sont l'amoxicilline (United States Pharmacopoeia standard lot $\mathrm{N}^{\circ}$ L0K369), la cloxacilline sodium (European Pharmacopoeia lot $\mathrm{N}^{\circ} 3 \mathrm{a}$ ) et le flucanozole. Toutes ces molécules ont été dissoutes dans du DMSO à une concentration de $6 \mathrm{mg} / \mathrm{mL}$.

\section{Détermination de l'activité antimicrobienne}

La méthode de diffusion sur disque a été utilisée pour tester les activités antibactériennes des différents extraits. Dans cette optique, des boites de Pétri contenant un milieu Mueller Hinton (MH) pour Staphylococcus aureus ATCC29213 et Sabouraud pour Candida albicans (Souche de laboratoire), ont été ensemencées avec un inoculum à $10^{5}$ UFC par écouvillonnage. Des disques stériles de $0,6 \mathrm{~cm}$ de diamètre sont ensuite placés sur la couche supérieure du milieu gélosé. Puis un volume de $20 \mu \mathrm{L}$ d'échantillon a été déposé sur chaque disque. Les boites ont ensuite été introduites dans l'étuve à $37^{\circ} \mathrm{C}$ pendant $24 \mathrm{~h}$ pour la souche bactérienne (Staphylococcus aureus) et 48 heures pour la souche fongique (Candida albicans). L'activité antimicrobienne est obtenue en mesurant le diamètre d'inhibition autour des disques (deux fois).

\section{Détermination de la Concentration Minimale Inhibitrice (CMI)}

La CMI, définie comme la plus faible concentration de l'échantillon qui inhibe la croissance visible d'un microbe, a été déterminée par la méthode de microdilution. Le milieu liquide utilisé est constitué de bouillon Mueller Hinton (M-H) pour Staphylococcus aureus ATCC29213 et de bouillon Sabouraud pour Candida albicans (Souche de laboratoire). La détermination de la CMI a été réalisée comme décrit ci-dessous. Dans chacun des puits de la plaque sont déposés $100 \mu \mathrm{L}$ de milieu de culture. Dans les puits de la première colonne sont déposés $100 \mu \mathrm{L}$ de chacune des solutions des produits à tester (extraits ou solutions d'antibiotique de référence). Les dilutions successives ont été faites à partir de cette première colonne, jusqu'à la dixième. Un 
volume de $10 \mu \mathrm{L}$ par puits d'une solution à $10^{5}$ UFC d'inoculum (microorganisme en solution dans le milieu de culture) est ensuite déposé dans tous les puits de la première à la onzième colonne. Les dix premières colonnes de la plaque servent donc à mesurer la CMI (Concentration Minimale Inhibitrice), la onzième colonne est un témoin de la croissance bactérienne, et la douzième, qui ne contient que du milieu de culture, un témoin pour vérifier la non-contamination du milieu. Puis sur chaque puits $10 \mu \mathrm{L}$ d'une suspension de culture bactérienne ou fongique à $10^{5}$ UFC a été déposée. A partir d'une solution mère à $6 \mathrm{mg} / \mathrm{mL}$ d'extraits ou d'antibiotique, de diméthyle sulfoxyde (DMSO), des gammes finales de concentrations d'essai allant de $3 \mathrm{mg} / \mathrm{mL}$ à 6 $\mu \mathrm{g} / \mathrm{mL}$ ont été préparées. Chaque microplaque a été couverte et incubée pendant 24 heures à $37^{\circ} \mathrm{C}$. Des puits avec bouillon sans extraits avec inoculum et des puits avec bouillon sans inoculum ont été utilisés comme contrôle positif et négatif. Une coloration limpide du puits a été interprétée comme l'absence de croissance et les puits montrant un aspect trouble ont été considérés comme positifs en raison de la croissance microbienne.

\section{Résultats}

\section{Diversité des espèces et modes d'administration}

Les plantes collécées comme intervenant dans le traitement des dermatoses sont au nombre de vingt-huit (28) (tableau 1). Elles appartiennent à vingt-six (26) genres et quatorze (14) familles. La famille des Fabaceae comportant six (6) genres et sept (7) espèces est la plus diversifiée. Viennent ensuite les familles des Malvaceae, Euphorbiaceae et des Combretaceae. Les genres les plus représentés, Senna et Jatropha comptent chacun deux espèces. Les plantes les plus couramment citées sont Jatropha chevalieri (28\%), Acacia seyal (15\%), Anogeissus leiocarpa (8,4\%), Stereospermum kunthianum (8\%), Sterculia setigera (10\%) et Leptadenia lancifolia (4\%) (tableau 1).

Tableau 1 : Liste des plantes utilisées contre les affections dermatologiques au Ferlo

\begin{tabular}{|c|c|c|c|c|c|c|}
\hline Famille & Espèce & Nom peul & $\begin{array}{l}\text { Nom } \\
\text { wolof }\end{array}$ & $\begin{array}{l}\text { Mode } \\
\text { d'administration }\end{array}$ & $\begin{array}{l}\text { Nombre } \\
\text { citations } \\
\text { (Peul) }\end{array}$ & $\begin{array}{l}\text { Nombre } \\
\text { citations } \\
\text { (Wolof) }\end{array}$ \\
\hline \multirow{2}{*}{ Anacardiacea } & \multirow[t]{2}{*}{$\begin{array}{l}\text { Anacardium } \\
\text { occidentale I }\end{array}$} & \multirow{2}{*}{ Darkasse } & \multirow[t]{2}{*}{ Darkasse } & $\begin{array}{l}\text { Application } \\
\text { locale }\end{array}$ & \multirow[t]{2}{*}{3} & \multirow[t]{2}{*}{1} \\
\hline & & & & Boisson & & \\
\hline \multirow{3}{*}{ Apocynaceae } & \multirow{3}{*}{$\begin{array}{l}\text { Leptadenia } \\
\text { lancifolia ( } \\
\text { Schumach. } \\
\text { \& Thonn.) } \\
\text { Decne. }\end{array}$} & \multirow{3}{*}{ Thiapatoki } & \multirow{3}{*}{ Tiakhat } & $\begin{array}{l}\text { Boisson } \\
\text { et bain }\end{array}$ & \multirow{3}{*}{15} & \multirow{3}{*}{1} \\
\hline & & & & $\begin{array}{l}\text { Boisson } \\
\text { et bain }\end{array}$ & & \\
\hline & & & & $\begin{array}{l}\text { Boisson } \\
\text { et bain }\end{array}$ & & \\
\hline
\end{tabular}




\begin{tabular}{|c|c|c|c|c|c|c|}
\hline & $\begin{array}{l}\text { Adenium } \\
\text { obesum (Fors } \\
\text { Roem. \& Sch }\end{array}$ & Darbogel & & Bain & 1 & \\
\hline Bignoniaceae & $\begin{array}{l}\text { Stereospermı } \\
\text { kunthianum } \\
\text { Cham. }\end{array}$ & Golôbi & Yeti deum & $\begin{array}{l}\text { Boisson } \\
\text { et bain }\end{array}$ & 23 & \\
\hline Capparaceae & $\begin{array}{l}\text { Capparis } \\
\text { tomentosa } \mathrm{La}\end{array}$ & $\begin{array}{l}\text { Gummi } \\
\text { Balewi }\end{array}$ & Khérégn & $\begin{array}{l}\text { Boisson } \\
\text { et bain }\end{array}$ & 1 & \\
\hline \multirow{6}{*}{ Combretaceae } & \multirow{3}{*}{$\begin{array}{l}\text { Anogeissus } \\
\text { leiocarpa (D) } \\
\text { Guill. \& Perr }\end{array}$} & \multirow{3}{*}{ Kodioli } & \multirow{3}{*}{ Ngédian } & $\begin{array}{l}\text { Boisson } \\
\text { et bain }\end{array}$ & \multirow{3}{*}{20} & \multirow{3}{*}{6} \\
\hline & & & & $\begin{array}{l}\text { Boisson } \\
\text { et bain }\end{array}$ & & \\
\hline & & & & Boisson & & \\
\hline & \multirow{2}{*}{$\begin{array}{l}\text { Guiera } \\
\text { senegalensis } \\
\text { J F. Gmel }\end{array}$} & \multirow{2}{*}{ Géloki } & \multirow{2}{*}{ Ngèr } & $\begin{array}{l}\text { Boisson } \\
\text { et bain }\end{array}$ & \multirow{2}{*}{4} & \\
\hline & & & & $\begin{array}{l}\text { Boisson } \\
\text { et bain }\end{array}$ & & \\
\hline & $\begin{array}{l}\text { Terminalia } \\
\text { avicennioides } \\
\text { Guill \& Perr }\end{array}$ & Pulemi & & Boisson & 1 & \\
\hline Cucurbitaceat & $\begin{array}{l}\text { Momordica } \\
\text { charantia L. }\end{array}$ & Borbof & Mbeurbeuf & $\begin{array}{l}\text { Boisson } \\
\text { et bain }\end{array}$ & 4 & 2 \\
\hline \multirow{6}{*}{ Euphorbiacea } & \multirow{2}{*}{$\begin{array}{l}\text { Euphorbia } \\
\text { balsamifera } \\
\text { Aiton }\end{array}$} & \multirow[t]{2}{*}{ Téyedoumwc } & \multirow{2}{*}{ Salan } & $\begin{array}{l}\text { Application } \\
\text { locale }\end{array}$ & \multirow{2}{*}{2} & \\
\hline & & & & $\begin{array}{l}\text { Boisson } \\
\text { et bain }\end{array}$ & & \\
\hline & \multirow{3}{*}{$\begin{array}{l}\text { Jatropha } \\
\text { chevalieri Be }\end{array}$} & \multirow{3}{*}{ Kolle dieri } & \multirow{3}{*}{$\begin{array}{l}\text { Viten } \\
\text { u beut }\end{array}$} & $\begin{array}{l}\text { Application } \\
\text { locale }\end{array}$ & \multirow{3}{*}{83} & \multirow{3}{*}{1} \\
\hline & & & & $\begin{array}{l}\text { Boisson } \\
\text { et bain }\end{array}$ & & \\
\hline & & & & $\begin{array}{l}\text { Boisson } \\
\text { et bain }\end{array}$ & & \\
\hline & $\begin{array}{l}\text { Jatropha } \\
\text { curcas L. }\end{array}$ & Tananani & Tabanani & $\begin{array}{l}\text { Application } \\
\text { locale }\end{array}$ & 2 & 1 \\
\hline \multirow{6}{*}{ Fabaceae } & $\begin{array}{l}\text { Acacia } \\
\text { seyal Delile }\end{array}$ & Bulbi & Surur & $\begin{array}{l}\text { Boisson } \\
\text { et bain }\end{array}$ & 38 & 6 \\
\hline & $\begin{array}{l}\text { Bauhinia } \\
\text { rufescens LaI }\end{array}$ & Namari & Râda & $\begin{array}{l}\text { Boisson } \\
\text { et bain }\end{array}$ & 2 & \\
\hline & \multirow{2}{*}{$\begin{array}{l}\text { Dalbergia } \\
\text { melanoxylon } \\
\text { Guill. \& Perr }\end{array}$} & \multirow{2}{*}{ Dialambani } & \multirow[t]{2}{*}{ Dialamban } & $\begin{array}{l}\text { Boisson } \\
\text { et bain }\end{array}$ & 1 & \\
\hline & & & & Boisson & & \\
\hline & $\begin{array}{l}\text { Bauhinia } \\
\text { reticulata D }\end{array}$ & Barkedji & Ngigiss & $\begin{array}{l}\text { Boisson } \\
\text { et bain }\end{array}$ & 1 & \\
\hline & & Badulo & Laydur & $\begin{array}{l}\text { Boisson } \\
\text { et bain }\end{array}$ & 1 & \\
\hline
\end{tabular}




\section{Boisson}

italica Mill

.

Senna

occidentali

$s$ (L.) Link

Aljana ji

Bantemare

Boisson

et bain

1

1

Tamarindus indica $\mathrm{L}$.

Diadmi

Dakkar

Boisson

et bain

1

1

Application

Sterculia

setigera

Bobori

Mbèp

locale

Application

locale

Malvaceae

Grewia

bicolor

Kélli

Kèl

Boisson

1

Juss.

\begin{tabular}{|c|c|c|c|}
\hline $\begin{array}{l}\text { Waltheria } \\
\text { indica L. }\end{array}$ & Kafaki & $\begin{array}{l}\text { Matum } \\
\text { Kewel }\end{array}$ & Boisson \\
\hline
\end{tabular}

Rhamnaceae

Ziziphus

Willd.

Diabi

Fowrou

Dem buki

Boisson

et bain

Mitracarpus

hirtus (L.) D(

Sélut

Mitragyna

inermis.

Rubiaceae

\begin{tabular}{l|l} 
Rubiaceae & Ku \\
&
\end{tabular}

(Willd.)

Koyli

Ndatukan

Boisson

et bain

Boisson

et bain

Khos

Kuntze

Boisson

Boisson

et bain

Boisson

et bain

Cissus

Vitaceae

populnea Bakagni

Guill. \& Perr

Boisson

Balanites

Zygophyllace

aegyptiaca

Delile

Muthiteki

Sump

Boisson

et bain
2

1

14

4

1

1

2 1

\begin{tabular}{l|l}
$14 \quad 8$
\end{tabular}


L'administration se fait essentiellement par voie orale et en application locale par bain (tableau 1). L'application directe sans aucune préparation concerne le latex. Beaucoup d'associations de plantes ont été proposées. Le nombre de plantes proposées par les informateurs varie d'un enquêté à un autre mais les procédés ne varient pas tellement. La plupart des enquêtés mentionnent deux à trois espèces mais certains vont jusqu'à cinq espèces. D'ailleurs, il existe une idée reçue au Ferlo selon laquelle toute association de sept racines de plantes différentes en décoction ou en macération soigne toujours.

\section{Niveaux de popularité des espèces proposées}

Près de la moitié des espèces utilisées (Leptadenia lancifolia, Stereospermum kunthianum, Capparis tomentosa. Euphorbia balsamifera, Bauhinia rufescens, Senna italica, Senna occidentalis, Sterculia setigera et Mitracarpus hirtus) sont exclusivement utilisées contre les dermatoses (IF= 100) (figure 2). Nous avons ensuite des espèces telles que Anogeissus leiocarpa, Jatropha curcas, Mitragyna inermis et Cissus populnea (IF variant entre 50 et 80) qui sont fréquemment utilisées dans le traitement des dermatoses. Les espèces très peu exploitées sont: Guiera senegalensis, Bauhinia rufescens, Tamarindus indica, Balanites aegyptiaca, Grewia bicolor, et Salvadora persica (figure 2).



Figure 2 : Niveaux de popularité (exclusif, fréquent, occasionnel) des plantes utilisées contre les dermatoses au Ferlo.

\section{Parties de plantes utilisées et leurs modes préparation}

Les organes utilisés vont des écorces (55\%) à la plante entière (1\%) en passant par la racine (5\%), la feuille (6\%), les rameaux (9\%) et le latex (24\%) (figure 3). 


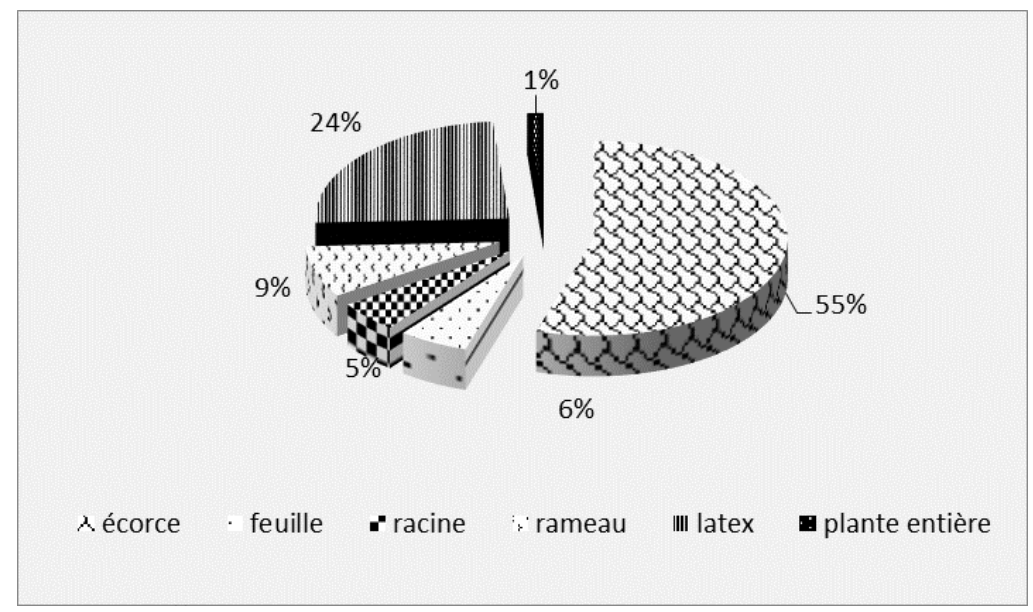

Figure 3 : Proportion des organes utilisés dans les affections dermatologiques au Ferlo

La uecuchuil (0<\%) esl ie illue ue piepaldivil ie pius couldill divis yue id macération et le broyage sont très peu usités $2 \%$ et $1 \%$ respectivement (figure 4). L’usage direct (35\%) sans aucune préparation particulière ne concerne que le latex.

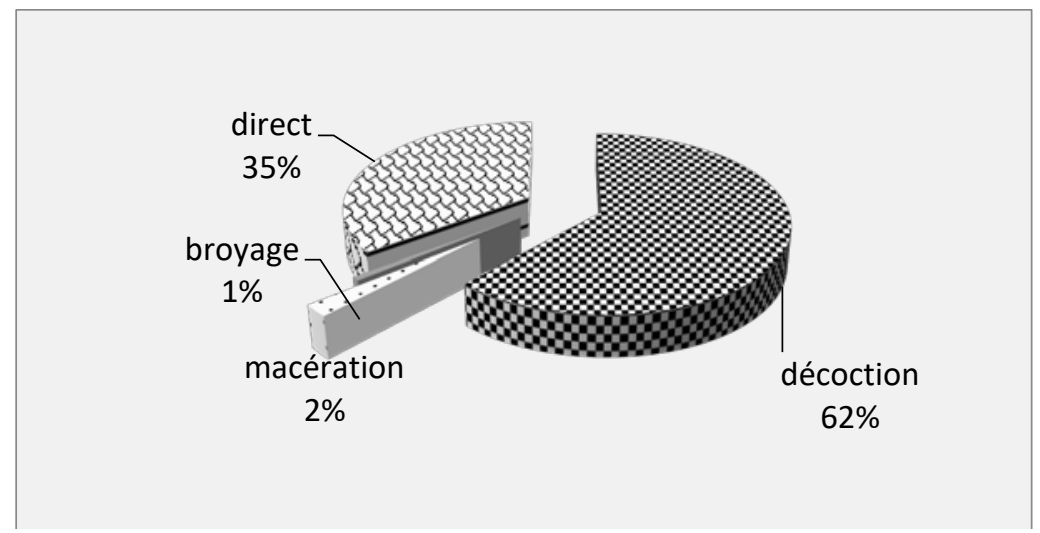

Figure 4: Proportion d'utilisation des modes de préparation des plantes utilisées dans le traitement des dermatoses au Ferlo

A noter que dans certains cas d'association de plantes, les premiers et deuxièmes décoctés sont souvent considérés comme trop forts pour être bus et ne doivent servir qu'au bain pour traiter les lésions cutanées superficielles. Seul le troisième décocté, plus léger est pris comme boisson. La posologie est de trois fois par jour tant pour la boisson (3 tasses) que pour le bain. Cependant, il serait préférable pour des raisons non évoquées de se baigner avant chaque repas et boire après les repas. 


\section{Diversité des plantes en fonction des localités visitées}

L'usage des plantes anti-dermatoses est très variable en fonction des localités (figure 5). La localité de Widou Thiengoli avec dix-neuf (19) espèces recensées, est celle où l'on a recueilli les plus grandes diversités. D'ailleurs, neuf (9) parmi ces espèces (Capparis tomentosa, Mitracarpus hirtus, Grewia bicolor, Guiera senegalensis, Euphorbia balsamifera, Salvadora persica, Anacardium occidentale, Dalbergia melanoxylon et Senna italica) ne sont exploitées qu'à Widou Thiengoli (figure 5). La localité de Widou Thiengoli est suivie par celle de Loughere Thiolly où l'on utilise douze (12) espèces et Barkédji en troisième position avec sept (7) espèces. Terminalia avicennioides n'est connue qu'à Loughere Thiolly et Tamarindus indica n’a été proposée qu'à Barkédji (figure 5). Les localités ayant proposé le moins d'espèces sont Amaly avec trois (3) espèces (Sterculia setigera, Jatropha chevalieri et Anogeissus leiocarpa) et Labgar avec 4 espèces (Anogeissus leiocarpa, Stereospermum kunthianum, Bauhinia rufescens et Momordica charantia) (figure 5). Anogeissus leiocarpa est utilisée dans toutes les localités excepté à Téssékéré Forage mais à des proportions inégales. Momordica charantia et Sterculia setigera sont également proposées, chacune dans quatre localités différentes (figure 5).

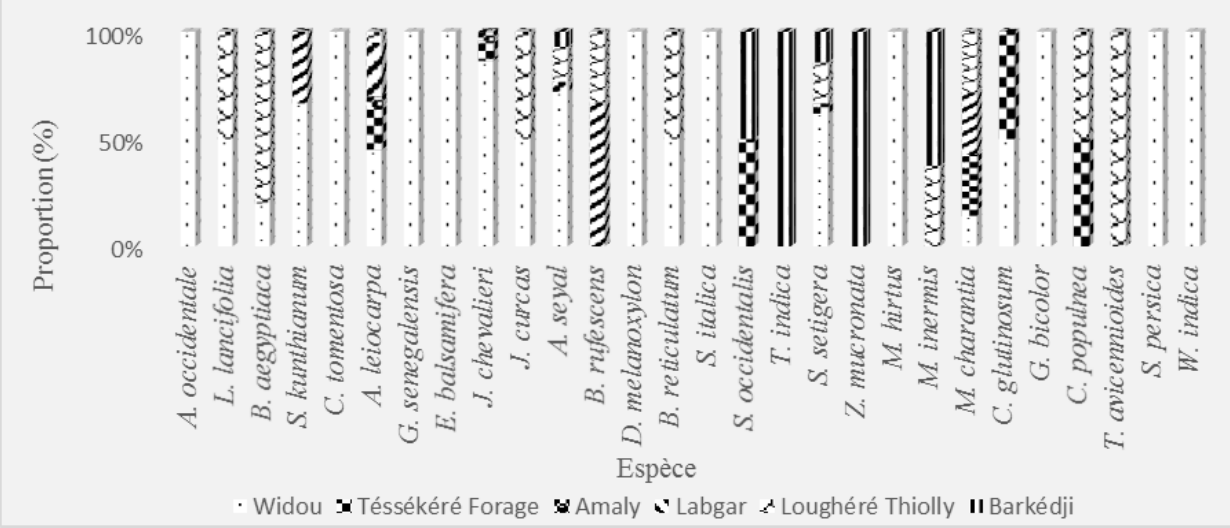

Figure 5 : Niveau d'utilisation des espèces utilisées contre les dermatoses en fonction des localités au Ferlo.

\section{Diversité et niveaux de fidélité des espèces entre Peul et Wolof}

La diversité spécifique exploitée par les Peul (28 espèces) est de loin supérieure à celle utilisée par les Wolof (11 espèces) (figure 6). Toutes les plantes utilisées par les Wolof le sont aussi par les Peul alors qu'il y a 16 espèces propres aux Peul. Chez ces derniers, treize (13) plantes sont exclusivement utilisées $(\mathrm{IF}=100)$ dans les dermatoses contre six (6) espèces chez les Wolof (figure 6). Pour toutes les espèces communes, 6 présentent le même niveau de fidélité chez les deux ethnies. L'usage de Jatropha curcas et d’Acacia seyal est plus connu chez les Peul. Cependant, Ziziphus mucronata, 
Bauhinia rufescens et Balanites aegyptiaca sont plus populaires chez les Wolof (figure 6) .



Figure 6 : Niveaux de fidélité des plantes antidermatoses chez les Peul et chez les Wolof.

\section{Croyances ou représentation}

Le Pityriasis versicolor communément appelé «xam » en Wolof et "lam lamé » en Peul, est une maladie dermatologique fréquente chez les personnes de teint clair. Selon les Peul, le Pityriasis versicolor est une atteinte bénigne qui ne mérite pas d''inquiètudes tant les enjeux dermatologiques sont négligeables et temporaires. Effectivement, beaucoup d'informateurs soulignent que le Pityriasis versicolor est une atteinte périodique ne survenant qu'en période d'hivernage. Certains affirment que cela n'arrive qu'aux jeunes et son apparition serait associée à la consommation abusive de lait. La plante la plus communément citée dans le traitement de Pityriasis versicolor est le Sterculia setigera appelée «Bobori » en Peul et «Mbeup » en Wolof. La couleur de l'écorce de la plante ressemble à celle des taches dues à la pathologie en question. D’où l'expression Peul : «Bobori woni diom lam lami » signifiant "Sterculia setigera est le propriétaire du Pitryasis versicolor ». C'est pourquoi, selon les croyances peules le traitement du Pityriasis versicolor avec Sterculia setigera exige la nudité du patient accompagnée d'incantations et un système d'échange entre la plante et le malade. Pour ce faire, il est recommandé de se mettre nu devant un Sterculia setigera, réciter quelques incantations, puis se frotter contre l'écorce poussiéreuse du tronc de la plante. Après, il faut jeter une pièce de cinq francs (5F cfa soit 0,008 euro) sous l'arbre en guise de paye, tourner le dos à la plante et s'en aller sans se retourner. Si jamais il arrivait par mégarde que le malade revienne sur ses pas aux abords de la plante, il recontacterait la maladie. Il convient par ailleurs de signaler le terme «thiadabe » en Peul et «ndoxum siti » en Wolof qui désigne un ensemble de pathologies dermatologiques sans 
distinction aucune. A chaque fois c'est une association de plantes qui est proposée pour le traitement.

\section{Activités antibactériennes et antifongiques des plantes les plus utilisées}

Les rendements d'extraction des plantes obtenus sont assez hétérogènes (tableau 2). Le rendement le plus élevé (14,5\%) a été enregistré pour l'extrait de Stereospermum kunthianum. Trois valeurs de rendements sont comprises entre 7\% et 5\%. Il s'agit de Mitragyna inermis (7\%), Anogeissus leiocarpa (6,5\%), Sterculia setigera (5,5\%) et Acacia seyal (5,5\%). Enfin, le plus faible rendement obtenu correspond à l'extrait de Leptadenia lancifolia. Parmi les sept (7) espèces sélectionnées, cinq ont présenté une activité contre les microorganismes testés (tableau 2). Sterculia setigera est la seule espèce ayant permis d'obtenir une CMI mesurable contre les deux souches étudiées, avec d'une part, une CMI de $3 \mathrm{mg} / \mathrm{mL}$ contre Staphylococcus aureus et d'autre part, une CMI de 0,755 mg/mL contre Candida albicans (tableau 2). Cette seconde mesure indique une activité antifongique modérée de l'extrait. Contre Staphylococcus aureus Acacia seyal et Anogeissus leiocarpa ont présenté une CMI mesurable aux valeurs de 3 et $1,5 \mathrm{mg} / \mathrm{mL}$ respectivement. Enfin Leptadenia lancifolia et Stereospermum kunthianum ont une CMI vis-à-vis de Candida albicans avec successivement des valeurs de CMI de 3 et $1,5 \mathrm{mg} / \mathrm{mL}$ (tableau 2).

Tableau 2 : Rendement d'extraction et activités antibactérienne et antifongique des plantes anti-dermatoses les plus utilisées au Ferlo

\begin{tabular}{|c|c|c|c|c|c|}
\hline \multirow[t]{2}{*}{ Souches microbienne } & \multirow{2}{*}{$\begin{array}{c}\text { Rendement } \\
\text { d'extraction } \\
\text { (\%) }\end{array}$} & \multicolumn{2}{|c|}{$\begin{array}{c}\text { Staphylococcus aureus } \\
\text { ATCC29213 }\end{array}$} & \multicolumn{2}{|c|}{$\begin{array}{c}\text { Candida albicans Souche de } \\
\text { laboratoire }\end{array}$} \\
\hline & & $\begin{array}{c}\text { Diamètre } \\
\text { d'inhibition (mm) }\end{array}$ & $\begin{array}{c}\mathrm{CMI} \\
(\mathrm{mg} / \mathrm{ml})\end{array}$ & $\begin{array}{l}\text { Diamètre d'inhibition } \\
\text { (mm) }\end{array}$ & $\begin{array}{c}\mathrm{CMI} \\
(\mathrm{mg} / \mathrm{ml})\end{array}$ \\
\hline $\begin{array}{c}\text { Leptadenia lancifolia } \\
\text { Vatke }\end{array}$ & 4 & & & & 1,5 \\
\hline $\begin{array}{c}\text { Jatropha chevalieri } \\
\text { Beille } \\
\end{array}$ & & & & & \\
\hline $\begin{array}{c}\text { Stereospermum } \\
\text { kunthianum Cham }\end{array}$ & 14,5 & & & 10 & 1,5 \\
\hline $\begin{array}{l}\text { Mitragyna inermis } \\
\text { Willd }\end{array}$ & 7 & & & & \\
\hline $\begin{array}{c}\text { Sterculia setigera } \\
\text { Delile }\end{array}$ & 5,5 & 10 & 3 & 10 & 0,755 \\
\hline Acacia seyal Del & 5,5 & 12 & 3 & & \\
\hline $\begin{array}{c}\text { Anogeissus leiocarpa } \\
\text { (DC.) Guill.\& Perr }\end{array}$ & 6,5 & 22 & 1,5 & 11 & \\
\hline Amoxicilline & & & 1,5 & & $22.10^{-3}$ \\
\hline Cloxacilline & & & 1,5 & & $16.10^{-3}$ \\
\hline Flucanazole & & & nd & & nd \\
\hline
\end{tabular}

nd : non déterminé 


\section{Discussion}

Sans distinction de la cause probable de la dermatose, beaucoup de remèdes servent à les traiter au Ferlo. On distingue les remèdes à base de plantes et les remèdes à base de produits transformés pas toujours végétaux. Au regard de nos résultats et de ceux de plusieurs auteurs (Diatta et al., 2013 et Cissé et al., 2012), il apparait que certaines familles botaniques (Euphorbiaceae, Apocynaceae, Rubiaceae, Fabaceae et Malvaceae) sont particulièrement impliquées dans le traitement des affections dermatologiques au Sénégal. Effectivement, il existe beaucoup d'espèces appartenant à ces familles citées dans le traitement des affections dermatologiques partout au Sénégal. Il s’agit de Mitracarpus hirtus, Jatropha curcas, Detarium microcarpum, Jatropha chevalieri, Cassia sieberiana, Leptadenia lancifolia et Waltheria indica (Diatta et al., 2013 et Cissé et al., 2012). Les Bainounk de Djibonker (région de Zinguinchor), utilisent 36 espèces (Diatta et al., 2013) alors que 27 plantes vendues dans les marchés de Dakar sont indiquées contre les affections dermatologiques (Cissé et al., 2012). La famille des Apocynaceae est la plus communément exploitée chez les Bainounk de (Diatta et al., 2013) tandis que les Fabaceae dominent au Ferlo tout comme à Dakar (Cissé et al., 2012). Au Ferlo, pour les espèces appartenant aux Euphorbiaceae et Apocynaceae, c'est le latex qui est plus utilisé et son usage est très souvent direct. Les Peul exploitent une plus grande diversité végétale que les Wolof. Cette maîtrise de l'usage des plantes locales pousse certains Peuls à régir la connaissance des plantes en marqueur ethnique de la "poularité » au mépris des Wolof et Maure, présents sur le territoire (Guissé et al., 2013). La maîtrise des savoirs spécifiques apparaît ainsi tel un emblème parmi tant d'autres de l'appartenance collective (Bromberger, 1986). L'acquisition des connaissances thérapeutiques des végétaux serait édifiée chez les Peul comme un « savoir-pouvoir » apportant à leur détenteur une fonction de choix au sein de la société (Guissé et al., 2012). En réalité, le mode de vie transhumant des Peul leur offre une plus grande chance de rencontrer des espèces nouvelles et d'échanger avec d'autres tradithérapeutes détenteurs de connaissances liées à ces plantes.

Au Ferlo, l'écorce est l’organe de la plante le plus souvent exploité suivi du latex alors que la décoction est le mode de préparation le plus commun puis l'usage direct. A contrario, Diatta et al. (2013) et Cissé et al. (2012) montrent que les populations de Dakar et de Djibonker (Ziguinchor) font plus recours respectivement aux feuilles et aux racines dans le traitement des affections dermatologiques. La fréquence d'utilisation des feuilles d'après Bitsindou (1986) serait due à leur accessibilité. Certaines plantes du Ferlo impliquées dans le traitement des pathologies dermatologiques ont les mêmes indications au Sud du pays (Ziguinchor). En effet, dans cette zone géographique, la macération des tiges épluchées de Jatropha curcas sont 
respectivement recommandés en boisson et en bain alors que les feuilles de Senna tora et de Senna occidentalis sont à frotter sur les zones atteintes (Sambou, 1998). De la même manière, certaines de ces espèces (Guiera senegalensis et Mitragyna inermis) sont impliquées dans le traitement des affections de la peau au Burkina Faso (Dakuyo, 2010). Nos résultats divergent avec ceux de Mozouloua et al. (2011) qui révèlent que les feuilles sont les organes végétaux les plus proposés dans le traitement des dermatoses à Bangui confortant ainsi Guèye et al., (2012) dont l'étude montre que les feuilles suivies des racines sont plus exploitées pour traiter la constipation. Parallèlement, Teklehaymanot (2009) et Sani (2009) indiquent que les racines et les écorces constituent les organes les plus proposés en médicine traditionnelle. Globalement, au Ferlo, le mode d'administration se fait par voie orale ou directement par le bain après décoction. En revanche chez les Bainounk, il est noté une nette prédominance de la macération (Diatta et al., 2013). Chez Guèye (2012) la macération est le mode de préparation le plus fréquent à Tomboronkoto (Sénégal oriental) alors qu'elle est un des procédés les moins évoqués au Ferlo. Des associations de plusieurs plantes dont les organes utilisés et les modes de préparation changent, sont très souvent recommandées. L'usage de combinaison de plantes pour le traitement de multiples affections, notamment dans le cas des infections microbiennes est par ailleurs une pratique courante dans de nombreuses cultures (Roumy et al., 2020 ; Gertsch, 2011; Li et al., 2011; Vigneron et al., 2005). Une combinaison de plantes a effectivement été mentionnée comme étant une pratique courante en Amazonie et ailleurs pour le traitement du paludisme (Vigneron et al., 2005). L' association de végétaux présente selon certains chercheurs, un intérêt en thérapie. En effet, Chenniappan et Kadarkarai (2010) soutiennent que les associations de plantes peuvent augmenter les effets antipaludiques des principes actifs présents dans les deux ou plusieurs espèces végétales utilisées. Wagner (2011) propose, de ce fait, au regard de l'usage archaïque récurrent des combinaisons thérapeutiques à base de végétaux en médecine traditionnelle, de trouver une preuve de la supériorité pharmacologique et thérapeutique de ces combinaisons comparées aux constituants isolés. D'ailleurs, l'utilisation de combinaisons de médicaments ne se limite pas aux plantes. Des combinaisons médicamenteuses sont effectivement indiquées pour les chimiothérapie anticancéreuse, de la tuberculose et de l'hypertension (Williamson, 2001). Les combinaisons de plantes au Ferlo sont rarement homogènes du point de vue de l'organe de la plante utilisé. En d'autres termes, les écorces d'une plante peuvent être associées au racines d'une autre plante et aux feuilles d'une dernière. D'une manière ou d'une autre Wagner (2011) et Vigneron et al. (2005) soutiennent qu'il y a souvent sous l'effet de la combinaison de plantes, une biodisponibilité améliorée et un effet cumulatif résultant des propriétés additives des espèces 
impliquées. Cuenca-Estrella (2004) et Jansen et al. (2009) admettent que cela serait d'une grande utilité médicale car, elle augmenterait l'efficacité thérapeutique, limiterait les effets secondaires et la nocivité des potions, ainsi que l'apparition de souches résistantes.

Les plantes ayant présenté une activité biologique dans le cadre de notre étude sont : Anogeissus leiocarpa, Acacia seyal qui sont antibactériennes et Leptadenia lancifolia, Stereospermum kunthianum qui sont anti candidale. Seule Sterculia setigera, a une activité à la fois antibactérienne et antifongique. De façon intéressante, cette plante possède par ailleurs une symbolique forte dans plusieurs régions du continent (Svetaz et al., 2010 ; Langewiesche et al., 2006). Du point de vue des croyances, les branches de Sterculia setigera étaient par ailleurs exploitées au Togo dans la confection de statuettes du fils aîné pour immortaliser leurs pères morts (Atakpama et al., 2012). La plante est également rapportée plusieurs fois comme «refuge de génies » dans la région de Baatombus à Pohunco au Nord-Bénin (Langewiesche et al., 2006). L'activité de Stereospermum kunthianum contre C. albicans $0,755 \mathrm{mg} / \mathrm{mL}$ est jugée modérée. Selon le critère proposé par Svetaz et al., (2010) d'une CIM $\leq$ $1000 \mu \mathrm{g} / \mathrm{mL}$ pour définir un extrait actif. Les fonctions curatives de Sterculia setigera en cas de boutons, de plaies ont été par ailleurs signalées par Lawal et $a l$. (2010) et Igoli et al. (2005). Certaines de ces espèces étudiées en tant que bâtonnets frotte-dents comptent parmi celles renfermant le plus de composés phytochimiques; il s'agit d'Anogeissus leiocarpa renfermant six (6) classes chimiques et comportant les meilleurs teneurs en alcaloïdes et en polyphénols puis Acacia seyal comportant cinq (5) classes chimiques (Diatta et al., 2021). De nombreuses molécules ont été isolées des extraits de Stereospermum kunthianum et des activités biologiques variées de l'espèce ont été mis en évidence (Oloche et al., 2016). Le travail de Aliyu et al. (2009) sur le Stereospermum kunthianum a montré une activité des feuilles de la plante avec une CMI de 2,09 mg/mL vis-à-vis de $S$. aureus. Les activités biologiques de Anogeissus leiocarpa et de Leptadenia lancifolia proposées dans le traitement des pathologies dermatologiques au Ferlo ont été confirmées par Mann et al. (2008) et Nikiema et al. (2001). Les racine d'Anogeissus leiocarpa sont efficaces contre diverses souches fongiques (A. niger, A. fumigatus, Penicillium spp., $M$. audouinii, T. rubrum) avec une gamme de CMI de $0,03 \mu \mathrm{g} / \mathrm{ml}$ à $0,06 \mu \mathrm{g} / \mathrm{mL}$ et des CMF (Concentrations Minimales Fongicides) comprises entre 0,04 et 0,07 $\mu \mathrm{g} / \mathrm{mL}$ (Mann et al., 2008). Ces valeurs démontrent des activités antimicrobiennes très fortes notamment vis-à-vis de Microsporum audouinii et de Trichophyton rubrum. L'extrait aqueux des tiges d'Anogeissus leiocarpa, utilisés au Ferlo comme bâtonnets frotte-dents élimine Porphyromonas gingivalis avec une CMI 128 $\mu \mathrm{g} / \mathrm{mL}, \quad$ C. albicans avec une CMI $16 \mu \mathrm{g} / \mathrm{mL}$ et Lactobacillus acidophilus avec une CMI $512 \mu \mathrm{g} / \mathrm{mL}$. Plusieurs triterpènes isolés à partir du latex de 
Leptadenia lancifolia au Burkina Faso ont été rapportés pour leur activité antiinflammatoire (Nikiema et al, 2001), en accord avec nos résultats sur l'usage du latex dans le traitement des affections dermatologiques au Ferlo. Le résultat de l'évaluation de l'activité antimycotique de Leptadenia lancifolia a montré que l'extrait de méthanol supprimait la croissance de Fusarium oxysporum et Aspergillus niger à $80 \mathrm{mg} / \mathrm{mL}$ avec des inhibitions allant de 59 à $73 \%$ (Aliero et $a l, 2009$ ). Les différences d'activités mesurées suivant les études pourraient être dues à diverses raisons, telles que les parties de la plante, le solvant d'extraction, le biotope de la plante et éventuellement le moment de la collecte. Malgré l'emploi actuel de plusieurs espèces de plantes en médecine traditionnelle contre les affections cutanées, très peu de recherches toxicologiques ont été réalisées et l'innocuité cutanée de beaucoup de traitements à base de végétaux demeure mal connue. D'ailleurs, Niang et al. (2015) rapportent que plusieurs atteintes dermatologiques découleraient ou seraient aggravées par l'usage inadéquat et exagéré de certaines espèces de plantes. Dans 56\% des cas, une dermatose existante a été accentuée par une automédication du malade avec des traitements végétaux (Niang et al., 2015). En effet, la plupart des patients de Dakar utilisant ces espèces ne les connaissent pas mais les achètent dans les marchés et les utilisent suite à des recommandations thérapeutiques. Les huiles essentielles, existantes dans plusieurs espèces de plantes, renferment des substances terpéniques capables d'occasionner des réponses allergènes ou irritantes cutanées (Niang et al., 2015). Les furanes sesquiterpènes et de triterpènes se rencontrent dans l'huile de Jatropha chevalieri ; les triterpènes sont également retrouvés dans les huiles de Leptadenia lancifolia, de Calotropis procera, de Stereospermum kunthianum, de Guiera senegalensis et de Detarium microcarpum (Reutemann et al., 2008; Nivosu et al., 2011). Les latex hautement cytotoxiques de Jatropha chevalieri, et Jatropha curcas sont connus pour provoquer une dermatite irritante (Baragay et al., 1998).

\section{Conclusion}

Au terme de cette étude, il apparaît que les Peul exploitent plus de plantes que les Wolof pour traiter les affections dermatologiques. La localité de Widou suivie de Loughéré Thiolly sont celles qui utilisent le plus d'espèces. Douze parmi les plantes recommandées ont un niveau de fidélité égaux à cent et sont donc exclusivement proposées dans le traitement des dermatoses. L'écorce est l'organe végétal le plus recommandé et la décoction le mode de préparation le plus courant. La plante la plus citées (Jatropha chevalieri) n’a présenté aucune activité vis-à-vis des deux souches microbiennes sélectionnées (S. aureus et C. albicans). En revanche, Sterculia setigera, dont l'usage fait intervenir un rituel, présente une activité élevée $(0,755 \mathrm{mg} / \mathrm{ml})$ vis-à-vis de Candida albicans . Quatre autres espèces Leptadenia lancifolia 
avec $1,5 \mathrm{mg} / \mathrm{ml}$ contre Candida albicans, Stereospermum kunthianum avec également 1,5 mg/ml contre Candida albicans , Acacia seyal avec $3 \mathrm{mg} / \mathrm{ml}$ contre Staphylococcus aureus et Anogeissus leiocarpa avec $1,5 \mathrm{mg} / \mathrm{ml}$ contre Staphylococcus aureus ont permis d'obtenir des concentrations minimales inhibitrices mesurables. Les effets synergiques supposés de l'association de plantes dans le traitement des dermatoses seraient considérés comme positifs. Des recherches menées sur les plantes utilisées contre les dermatoses au Sénégal ont montré que certaines plantes aggraveraient la pathologie. Dès lors, il urge de mener des études toxicologiques sur ces plantes pour identifier le ou les facteur (s) responsables de ces phénomènes.

\section{Remerciements :}

Ce travail a bénéficié d'une aide du Labex DRIIHM, programme « Investissements d'avenir » portant la référence ANR-11-LABX-0010.

\section{References:}

1. Aliero, AA \& Wara, SH. (2009). Validating the medicinal potential of Leptadenia hastate African Journal of Pharmacy and Pharmacology 3(6),335-338.

2. Aliyu, MS., Hanwa, UA., Tijjani, MB., Aliyu, AB \& Ya’u B. (2009) Phytochemical and antibacterial properties of leaf extract of Stereospermum kunthianum (Bignoniaceae). Nigerian J Basic and Applied Sci 17(2), 235-239.

3. ANSD. (2013). Recensement de Téssékéré de 2013, rapport final : 97p.

4. Arbonnier, M. (2002). Arbres, arbustes et lianes des zones d'Afrique de l'Ouest. Paris, CIRAD , 573 p.

5. Atakpama, W., Batawila, K., Dourma, M., Pereki, H., Wala, K., Dimobe, K., Akpagana, K, M \& Gbeassor M. (2012). Ethnobotanical Knowledge of Sterculia setigera Del. in the Sudanian Zone of Togo (West Africa) International Scholarly Research Notice.

6. Bakhoum, A. (2013). Dynamique des ressources fourragères : Indicateur de résilience des parcours communautaires de Téssékéré au Ferlo (Nord Sénégal). Thèse doct., Ecologie, UCAD.

7. Baraguey, C., Auvin, C., Blond, A., Cavalier, F., Lezenven, F ., Pousset, JL \& Bodo, Bl.(1998). Isolation and synthesis of chevalierins A, B and C, cyclic peptides from the latex of Jatropha chevalieri. $J$ Chem Soc Perkin Trans, 1(18),3033-9.

8. Begossi; A. (1996). Use of ecological methods in ethnobotany: Diversity indices. Ecological Methods in Ethnobotany, 50, 280-289.

9. Berhaut J. (1967). Flore du Sénégal plus complète avec les forêts humides de la Casamance. Ed. ClairAfrique, 485 p. 
10. Bitsindou, M. (1986). Enquête sur la phytothérapie traditionnelle à Kindamba et Odzala (Congo) et analyse de convergence d'usage de plantes médicinales en Afrique centrale. Mem. Doc (inéd) Univ. Libre de Bruxelles 482pp

11. Bromberger, C. (1986). Habitat, Architecture et Société Rurale dans la Plaine du Gilân (Iran septentrional). Paris, unEsCo. Version anglaise: Habitat, Architecture and Rural Society in the Gilân Plain (Northern Iran). Bonn, Ferd Dümmlers Verlag (bonner geographische abhandlungen, heft 80)

12. Chenniappan, K \& Kadarkarai, M. (2010). In vitro antimalarial activity of traditionally used Western Ghats plants from India and their interactions with chloroquine against chloroquine-resistant Plasmodium falciparum. Parasitology Research, 107(6), 1351-1364.

13. Cissé, A., Guèye, M., Koma, S \& Akpo, LE. (2012). La commercialisation des plantes utilisées contre les affections dermatologiques dans les marchés de Dakar et de sa banlieue. In Boëtsch G., Guerci A., Gueye L. \& Guissé A., 2012: Les plantes du Sahel, Ed. CNRS, Paris, pp 323-333.

14. Cisse, A., Guèye, M., Ka, A., Ndiaye, F., Koma, S \& Akpo, LE (2016). Ethnobotanique des plantes médicinales chez les bergers peuls de Widou Thiengoly de la commune de Téssékéré (Ferlo-Nord Sénégal). Journal of Applied Biosciences 98, 9301-9308

15. Cuenca-Estrella, M. (2004). Combinations of antifungal agents in therapy - what value are they? J Antimicrob Chemother 54 (5) : 854869.

16. Dakuyo, VM. (2010). Contribution à l'étude de la pharmacopée traditionnelle burkinabe : enquête ethnopharmacologique dans la région des cascades. Thèse de doctorat en Pharmacie UCAD.

17. Diatta, CD., Guèye, M. \& Akpo, LE. (2013). Les plantes médicinales utilisées contre les dermatoses dans la pharmacopée Baïnounk de Djibonker, région de Ziguinchor (Sénégal), Journal of Applied Biosciences, 70, 5599- 5607.

18. Diatta, BD., Houël, E., Niass, O., Guèye, M \& Boetsch G. (2019). Activités antimicrobiennes des plantes utilisées comme bâtonnets frotte-dents (cure-dents) par les Peul de la commune de Tessékéré (Ferlo Nord, Sénégal) International Journal of Biological and Chemical Sciences 13(3), 1444-1457

19. Diatta, BD., Niass, O., Diouf, M., Guèye, M., Houël, E \& Boetsch, G. (2021) . Diversité et composition phytochimique des bâtonnets frotte-dents (cure-dents) proposés chez les Peul de la commune de Tessekere (Ferlo, Nord Sénégal). Journal of Applied Biosciences, 158, 16267-16281. 
20. El Rhaffari, L., Zaid, A., Hammani, K. \& Benlyas M. (2002). Traitement de la leishmaniose cutanée par la phytothérapie au Tafilalet. Revue Biologie et Santé, 1 (4), 45-54.

21. Gertsch, J. (2011). Botanical drugs, synergy, and network pharmacology : Forth and back to intelligent mixtures. Planta Medica 77, 1086-1098.

22. Guèye, M. (2012). Etude ethnobotanique chez les Malinké de la Communauté rurale de Tomboronkoto (Région de Kédougou) et valorisation des collections historiques de l'herbier de l'Institut Fondamental d'Afrique Noire. Thèse de Doctorat d'État ès Sciences, UCAD.

23. Guèye, M., Cisse, A., Diatta, C D., Diop, S \& Koma, S. (2012). Etude ethnobotanique des plantes utilisées contre la constipation chez les Malinké de la communauté rurale de Tomboronkoto International Journal of Biological and Chemical Sciences 6 (2), 778-779

24. Guissé, A. (2013). "Portraits d’arbres" in Boetsch G., Spaini, A., 2013 La Grande Muraille Verte. Toulouse, Edition Privat 144 pages.

25. Houël, E., Rodrigues, AMS., Jahn-Oyac A., Bessière, JM., Eparvier, V., Deharo E \& Stien, D, (2014). In vitro antidermatophytic activity of Otacanthus azureus (Linden) Ronse essential oil alone and in combination with azoles. Journal of Applied Microbiology 116(2), 288-294.

26. Igoli, JO., Tsenongo, SN. \& Tor-Ayin, TA. (2011). A survey of antivenimous, toxic and other plants used in some parts of Tivland, Nigeria. International Journal of Medicinal and Aromatic Plants , 1(3), 240- 244

27. Jansen, G., Lee, AL., Epp, E., Fredette, A., Surprenant, J., Harcus, D., Scott M., Tan, E. \& Thomas, DY .(2009). Chemogenomic profiling predicts antifungal synergies. Molecular Systems Biology 5 (1) 338.

28. Langewiesche, K. (2006). La forêt, les ancêtres et le marché : Perceptions locales de la forêt et de ses changements au Nord-Bénin. Afrika Spectrum, 41(2): 221-248.

29. Lawal, HO., Chang, HY., Terrell, AN., Brooks, ES., Pulido, D., Simon, AF. \& Krantz, DE. (2010). The Drosophila vesicular monoamine transporter reduces pesticideinduced loss of dopaminergic neurons. Neurobiology of disease, 40(1),102-112.

30. Leonti, M., Casu, L., De Oliveira Martins, DT., Rodrigues, E \& Benitez, G. (2020). Ecological theories and major hypotheses in ethnobotany: their relevance for ethnopharmacology and pharmacognosy in the context of historical data. Revista Brasileira de Farmacognosia, 1-16. 
31. Leonti, M., Casu, L., De Oliveira Martins, DT., Rodrigues, E \& Benitez, G. (2020). Ecological Theories and Major Hypotheses in Ethnobotany: Their Relevance for Ethnopharmacology and Pharmacognosy in the Context of Historical Data. Revista Brasileira de Farmacognosia, 1-16.

32. Li, S., Zhang, B \& Zhang, N. (2011). Network target for screening synergistic drug combinations with application to traditional Chinese medicine. BMC Systems Biology 5(Suppl 1):S10.

33. Mann, A., Banso, A \& Clifford, LC. (2008). An antifungal property of crude plant extracts from Anogeissus leiocarpus and Terminalia avicennioides Tanzania Journal of Health Research 10 (1), 34-38.

34. Miehe, M. (2007). Continuous monitoring of vegetation in the experimental perimeter at Widou Thiengoly within the framework of Senegalese-German projects, 1981-2007. Experimental set-up, monitoring methods and prospects for evaluation, Workshop on the transfer of the scientific heritage of PAPF, Dakar, $26 \mathrm{p}$.

35. Mozouloua, D., Apema, AKR \& Nguengue, JP. (2011). Etude préliminaire des plantes à effet antidermatosiques utilisées en pharmacopé à Bangui URSAD, 3, 5-6.

36. Newmann, DJ \& Cragg, JM. (2007). Natural products as sources of new drugs over the last 25 years. Journal of Natural Products 70 (3), 461-467.

37. Ndong, AT., Ndiaye, O., Sagna, M B., Diallo, A., Galop, D \& Guissé, A. (2015). Caracterisation de la vegetation ligneuse sahelienne du Senegal: cas du Ferlo. International Journal of Biological and Chemical Sciences, 9(6), 2582-2594.

38. Niang, K., Ndiaye, O., Diallo, A \& Guisse, A. (2014). Flore et structure de la végétation ligneuse le long de la Grande Muraille Verte au Ferlo, Nord Sénégal Journal of Applied Biosciences 79, 6938 - 6946.

39. Niang, SO., Tine, Y., Diatta, BA., Diallo, M., Fall, M., Seck, NB \& Kane A. (2015). Negative cutaneous effects of medicinal plants in Senegal British Journal of Dermatology,173,26-29.

40. Niass, O., Sarr, SO., Diop, A., Diop, A., \& Diop, YM. (2015). Etude in vitro de l'activité antibactérienne de quelques plantes utilisées en médecine traditionnelle locale au Saloum (Sénégal). La Science en Liberté, 7(150801), 1-10.

41. Nikiéma, J B., Fastré, R V., Vanhaelen, M., Fontaine, J., Graef, CD \& Heenen, M. (2001). Effects of anti inflammatory triterpenes isolated from Leptadenia hastata latex on keratinocyte proliferation. Phytotherapy Research, 15(2): 131-134.

42. Nivosu, CO., Yahayah, K \& Igbkwe, IO. (2011). Toxicity and anticoccidial efficacy of some plants used in the traditional treatment 
of avian coccidiosis in semi-arid north eastern Nigeria. Research Journal of Parasitology, 6 (1),18-30.

43. Oloche, JJ., Okwuasaba, JF \& Obochi, GO. (2015). Review of Phytochemical, Pharmacological and Toxicological Profile of Stereospermum kunthianum. Journal of Advances in Medical and Pharmaceutical Sciences, 5(1), 1-10.

44. Redding, S., Smith, J., Farinacci, G., Rinaldi, M., Fothergill, A., Rhine-Chalberg, J \& Pfaller, M. (1994). Resistance of Candida albicans to fluconazole during treatment of oropharyngeal candidiasis in a patient with AIDS: documentation by in vitro susceptibility testing and DNA subtype analysis. Clinical Infectious Diseases, 18(2) : 240242.

45. Reutemann, P \& Ehrlich, A. (2008). Neem oil: an herbal therapy for alopecia causes dermatitis. Dermatitis: contact, atopic, occupational, drug, 19(3), E12-15.

46. Rios, JL \& Recio, MC. (2005). Medicinal plants and antimicrobial activity. Journal of Ethnopharmacoly 100 (1-2), 80-84.

47. Rodrigues, AMS., Eparvier, V., Odonne, G., Amusant, N., Stien, D \& Houël, E. (2019). The antifungal potential of (Z)-ligustilide and the protective effect of eugenol demonstrated by a chemometric approach. Scientific Reports 9(1), 1-9.

48. Roumy, V., Ruiz Macedo, JC., Bonneau, N., Samaillie, J., Azaroual, N., Encinas, LA., Rivière, C., Hennebelle, T., Sahpaz, S., Antherieu, S., Pinçon, C., Neut, C., Siah, A., Gutierrez-Choquevilca, AL \& Ruiz L. (2020). Plant therapy in the Peruvian Amazon (Loreto) in case of infectious diseases and its antimicrobial evaluation. Journal of Ethnopharmacology 249, 112411.

49. Sambou, S. (1998). Enquête ethno pharmacologique en milieu Diola (Casamance) : Examen de 78 plantes médicinales sénégalaises utilisées dans la thérapeutique des plaies et brûlures. Thèse de doctorat en pharmacie, UCAD.

50. Sani, RA. (2009). Caractérisation biophysique des ressources ligneuses dans un site reverdi et un site dégradé dans le département de Mirriah. Mémoire de fin de cycle en vue de l'obtention du diplôme d'ingénieur, option: eaux et forêts. Université Abdou Moumouni (Niger).

51. Senghor, S. (2008). Contribution à la pharmacopée traditionnelle des Diola blouf Enquête ethnobopharmacologique dans le département de Bignona. Thèse de doctorat en Pharmacie UCAD

52. Sreekumar, O \& Hosono, A. (1998). The antimutagenic properties of a polysaccharide produced by Bifidobacterium longum and its cultured 
milk against some heterocyclic amines. Canadian Journal of Microbiology, 44(11),1029-1036.

53. Svetaz, L., Zuljan, F., Derita, M., Petenatti, E., Tamayos, G., Caceres, A., Cechinel Filho, V., Gimenez, A., Pinzon, R., Zacchino, S. \& Gupta MP. (2010). Value of the ethnomedical information for the discovery of plants with antifungal properties. A survey among seven Latin American countries. Journal of Ethnopharmacology. 127(1), 137-158.

54. Teklehaymanot, T. (2009). Ethnobotanical study of knowledge and medicinal plants used by the people in Dek Islan in Ethiopia. Journal of ethnopharmacology, 124 (1), 69-78.

55. Togola, A., Diallo, D., Dembele, S., Barsett, H. \& Paulsen, BS. (2005). Ethnopharmacological survey of different uses of seven medicinal plants from Mali, (West Africa) in the regions Doila, Kolokani and Siby. Journal of Ethnobiology and Ethnomedicine, 1 (7), 1-9.

56. Vigneron, M., Deparis, X., Deharo, E. \& Bourdy, G. (2005). Antimalarial remedies in French Guiana: A knowledge attitudes and practices study. Journal of Ethnopharmacol. 98(3) 351-360.

57. Wagner, H. (2011). 'Synergy research: Approaching a new generation of phytopharmaceuticals', Fitoterapia, 82 (1), 34-37.

58. Williamson, EM. (2001). 'Synergy and other interactions in phytomedicines', Phytomedicine, 8(5), 401-409. 